\title{
Post-operative renal failure management in mechanical circulatory support patients
}

\author{
Danielle Austin $^{1,2}$, Peter McCanny ${ }^{1,2}$, Anders Aneman ${ }^{1,2,3}$ \\ ${ }^{1}$ Intensive Care Unit, Liverpool Hospital, South Western Sydney Local Health District, Sydney, Australia; ${ }^{2}$ South Western Sydney Clinical School, \\ University of New South Wales, Sydney, Australia; ${ }^{3}$ Faculty of Medicine and Health Sciences, Macquarie University, Sydney, Australia \\ Contributions: (I) Conception and design: All authors; (II) Administrative support: None; (III) Provision of study materials: All authors; (IV) Collection \\ and assembly of data: All authors; (V) Data analysis and interpretation: All authors; (VI) Manuscript writing: All authors; (VII) Final approval of \\ manuscript: All authors. \\ Correspondence to: Dr. Danielle Austin, MBBS, FRACP, FCICM. Staff Specialist, Liverpool ICU, SWSLHD, Conjoint Lecturer SWS Clinical School, \\ UNSW, Australia. Email: danielle.austin@health.nsw.gov.au.
}

\begin{abstract}
Acute kidney injury (AKI) occurs commonly in patients requiring mechanical circulatory support (MCS) after cardiothoracic surgery. The prognostic implications of AKI in this patient group relate closely to the pathophysiology and risk factors associated with the underlying disease; pre-operative, intra-operative, and post-operative variables; hemodynamic factors; and type of support device used. General approaches to AKI management, including prevention strategies, medical management, and hemodynamic support, are also applicable in patients requiring MCS. Approaches to renal replacement therapy vary depend on patient factors, device-specific factors, and local preferences and experience. In this invited narrative review, we discuss the pathophysiology, risk factors, and prognostic implications of AKI in post-operative adult patients following institution of MCS. Management strategies for AKI are presented with a focus on those supported with either extracorporeal membrane oxygenation or a ventricular assist device.
\end{abstract}

Keywords: Mechanical circulatory support (MCS); acute kidney injury (AKI); extracorporeal membrane oxygenation (ECMO); left ventricular assist device (LVAD)

Submitted Feb 02, 2020. Accepted for publication Apr 30, 2020.

doi: $10.21037 /$ atm-20-1172

View this article at: http://dx.doi.org/10.21037/atm-20-1172

\section{Introduction}

This invited narrative examines the pathophysiology and risk factors for the development of acute kidney injury (AKI) in adult patients following institution of mechanical circulatory support (MCS) (Table S1), with a focus on extracorporeal membrane oxygenation (ECMO) and ventricular assist devices (VAD). The evidence concerning prognostic implications for mortality and recovery of renal function are outlined. Evidence-based management strategies to treat post-operative MCS patients with AKI are suggested including medical management, hemodynamic support, and principles and strategies for the provision of dialysis. Pediatric cases and MCS instituted in a nonoperative context are not considered.
Refractory cardiogenic shock requiring MCS affects between $0.4 \%$ and $3.7 \%$ of patients after cardiac surgery (1). Post-cardiotomy cardiogenic shock (PCCS) is an increasing indication for the use of ECMO, however the expansion in ECMO use does not appear to have correlated with improved outcomes (2), and the use of ECMO in this setting carries a relatively high mortality risk (3). AKI is common during ECMO overall with several studies reporting an incidence between $70-85 \%$ that is associated with significantly increased mortality rates up to $80 \%$ (4-7). In the setting of severe AKI requiring renal replacement therapy (RRT), the mortality may be increased up to 4-fold (8) with an overall 90-day survival reduced to $17 \%$ (9).

Among patients who receive durable support in the form of a VAD, mild-to-moderate renal dysfunction is common in 
the pre-operative phase as a result of chronic heart failure. The relationship between renal dysfunction and prognosis is more complex in these cases, where the association between pre-existing renal failure and poor outcomes has important implications for case selection but evidence from multiple investigators has yielded mixed results. The development of new AKI following VAD implantation appears lower compared to ECMO with a reported incidence between 11-45\% (10), albeit some studies have found an incidence of $70 \%$, similar to ECMO (11). Post-VAD implantation AKI has strong negative prognostic implications for survival, quality of life and ability to bridge to transplant.

The presence of AKI in patients on MCS is often the result of progression from the condition that precipitated ECMO/VAD support. The complex interplay between patient factors including pre-morbid risks, pre-MCS and intra-MCS treatment factors remains incompletely investigated and understood which underscores the potential to avoid iatrogenic harm as well as to optimize therapeutic benefit.

\section{Pathophysiology}

Renal function is dependent on adequate perfusion pressure, as determined by the difference between mean arterial pressure and central venous pressure, oxygen delivery, autoregulatory capacity including the partition of blood flow between the renal cortex and medulla, the resistance ratio between efferent and afferent glomerular vessels and tubuloglomerular feedback. The kidneys receive about $20 \%$ of cardiac output and active transport processes for sodium, water, glucose and amino acids in the proximal tubules represent approximately $80 \%$ of renal oxygen consumption (12). In patients progressing to veno-arterial ECMO (VA-ECMO) post-cardiotomy or selected for VAD implantation, renal homeostasis has typically been disrupted for prolonged periods of time (8) and multiple, complex, interacting and sometimes synergistic pathophysiological factors contribute within variable time frames to AKI (13) (Figure 1). In this view, AKI is an epiphenomenon to MCS. Arterial hypotension, increased venous pressures from fluid overload and high intrathoracic pressures during ventilation associated with increased right ventricular afterload, a low cardiac output state and insufficient oxygen delivery, neurohumoral activation with blood flow diverted away from splanchnic organs and potentially aggravated by exogenous vasopressors are all established in the generic pathophysiology of AKI (14). While the importance of these macrocirculatory derangements may be obvious, changes to the microcirculation, i.e., the glomerular and peritubular vascular networks, appear even more important (15). The renal microcirculation in AKI is characterized by reduced and heterogeneous perfusion with the development of ischemic, hypoxic areas adjacent to zones with normal oxygenation, particularly in the outer medulla (16) that stimulate the excessive production of reactive oxygen species (17). Increased endothelial permeability as a result of degradation of the glycocalyx (18) results in interstitial edema leading to an increased oxygenation distance and increased pressure within the renal capsule that further compromises renal perfusion. Endothelial dysfunction is both a trigger and a consequence of a systemic inflammatory response, involving activated leukocytes and the release of proinflammatory cytokines as well as the formation of microthrombi (19). A dysregulated inflammatory response is also associated with a mismatch of macro- to microvascular flow distribution in the presence of a hyperdynamic, vasoplegic hemodynamic state that is conducive to reduced glomerular filtration pressure and impaired renal autoregulation (20).

The pathophysiology outlined above is operative both before and during MCS. In post-cardiotomy VA-ECMO patients and during VAD implantation, use of cardiopulmonary bypass triggers many of the pathophysiological pathways (21) and surgery may further contribute to AKI by excessive bleeding and need for massive transfusion (22). During MCS, the interfaces between blood and the non-endothelialized ECMO circuit as well as between air and blood in the oxygenator may elicit coagulopathy, bleeding and formation of microthrombi and inflammation, hyperpermeable capillaries and vasoplegia. The latter is most frequently observed in left ventricular assist device (LVAD) patients (23).

A particular consideration in the peri-commencement period of MCS is ischemia-reperfusion injury. Expansion of the intravascular volume, administration of vasopressors and inotropes, and lung protective ventilation represent common interventions in the pre-MCS period. Intervening administration of diuretics, weaning attempts of vasoactive support and high-PEEP strategies are also common. These interventions, aimed to delay or obviate the need for MCS with preservation of renal function less of a priority, may result in periods of ischemia and reperfusion. Once MCS has been established, the preload-dependent characteristics of centrifugal MCS leads to further fluid loading with 


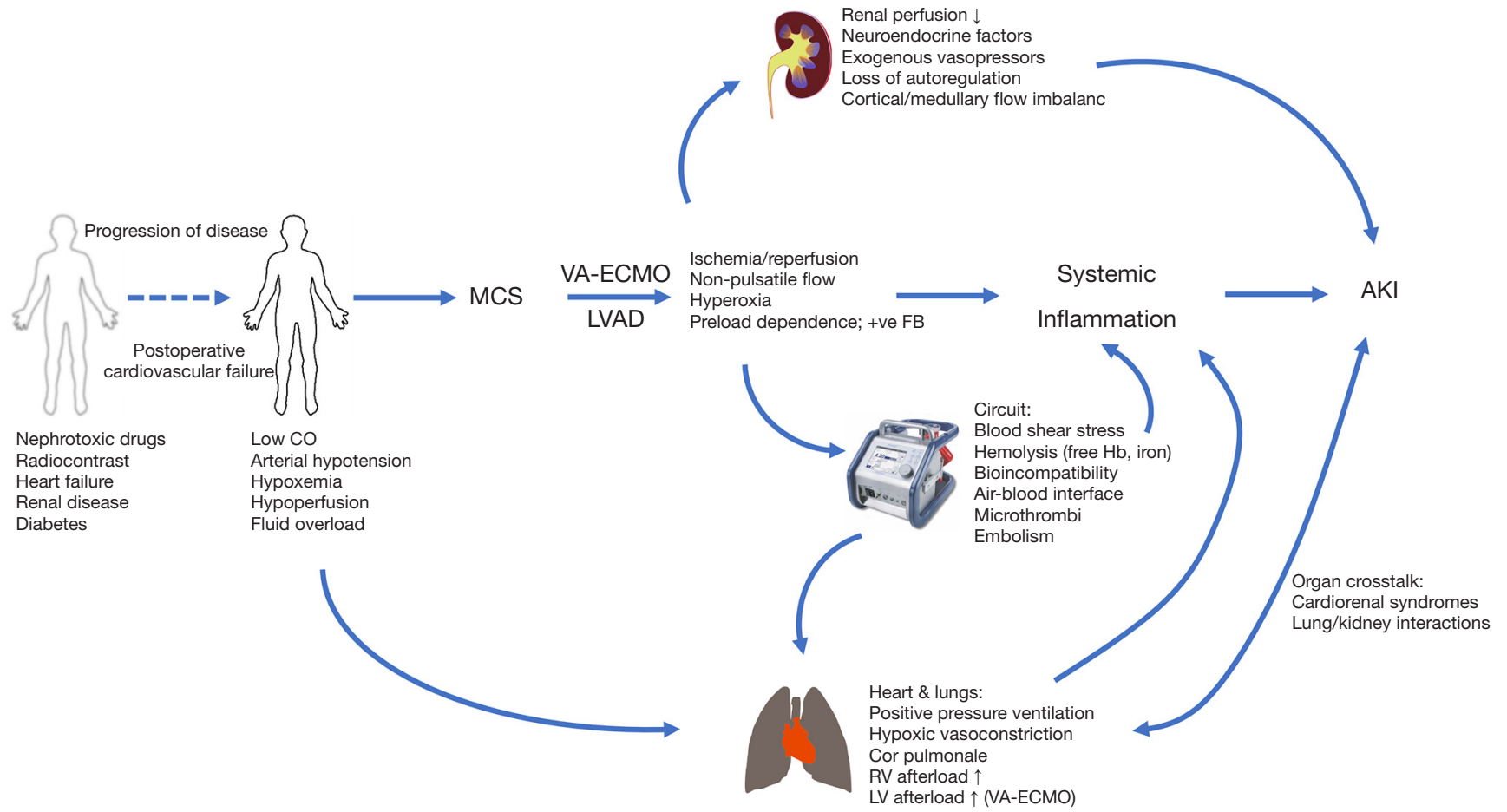

Figure 1 Pathophysiological pathways for acute kidney injury in patients following implantation of mechanical circulatory support.

typically frequent initial changes to MCS circuit pressures and flows, with reduced ventilatory support. This leads to increased systemic perfusion and markedly elevated oxygenation on VA-ECMO that again may lead to episodes of ischemia and reperfusion. The microcirculatory effects of ischemia-reperfusion have been linked to AKI $(24,25)$ but the importance of timing and delivery of MCS in this context is unclear. Factors related to the MCS circuit can lead to increased shear stress, high circuit pressure and minimal or even no arterial pulsatility. This may further compromise renal function by hemolysis and the release of free hemoglobin and free iron generation, or subhemolytic erythrocyte deformation leading to reduced oxygen carrying capacity $(13,26)$. In VA-ECMO patients with femoral cannulation, failure to establish adequate antegrade arterial perfusion may lead to compartment syndrome with myoglobinuria and AKI.

\section{Diagnosis of AKI}

A consensus definition of AKI has been formulated within the Acute Dialysis Quality Initiative with the original RIFLE (Risk, Injury, Failure, Loss of function, End stage failure) and revised AKIN (Acute Kidney Injury Network) criteria consolidated into the KDIGO (Kidney Disease Improving Global Outcomes) definition (27). These criteria all rely on serum creatinine, estimated glomerular filtration rates (GFR) and urine output while metabolic acidosis, electrolyte abnormalities and fluid overload are other important facets of AKI in MCS patients. Neither creatinine nor urine output can be used as concurrent markers of renal function and significant reductions in GFR are typically necessary before RIFLE or AKIN criteria are met. The diagnostic precision of creatinine in patients considered for MCS may be further confounded by pre-existing catabolism, sarcopenia, volume overload and positive fluid balance, leading to spuriously low creatinine levels and potentially underestimating the true extent of renal dysfunction. Many studies of AKI in MCS populations have utilized the requirement for RRT as a crude, dichotomized diagnosis of AKI given their retrospective designs.

A retrospective review of 67 patients supported with VA-ECMO for PCCS found that both RIFLE-failure and AKIN stage 3 within the initial 48 hours of VA-ECMO were independently associated with hospital mortality (28) without predictive superiority of either system. The KDIGO criteria generated relatively higher discriminatory power for AKI to predict in-hospital mortality compared to 
the RIFLE and AKIN systems in 167 patients undergoing VA-ECMO support (29).

In an analysis of 4836 consecutive patients undergoing cardiac surgery with cardiopulmonary bypass, more patients were diagnosed with AKI using AKIN criteria compared to RIFLE criteria with a higher odds ratio for mortality up to 30 days for the former (RIFLE $5.3 v s$. AKIN 4.5) (30). The variable performance of AKI diagnostic systems and limitations of creatinine, GFR and urine output as endpoints have stimulated interest in alternative assessments of renal function, including an array of novel biomarkers measurable in plasma and/or urine. In critical illness, neutrophil gelatinase-associated lipocalin (NGAL) and cystatin $\mathrm{C}$ have been the most studied of these, but there is insufficient evidence overall, and certainly in patients on MCS, to establish whether these biomarkers might detect renal dysfunction earlier and with greater precision to allow more timely prevention or management of AKI (31).

\section{Risk factors and prognostic implications}

The identification of risk factors for AKI in patients receiving MCS has been established through extrapolation of evidence in patients undergoing cardiopulmonary bypass (CPB) as well as from clinical case series and database reviews using mainly retrospective, observational methodology. In general, risk factors may be categorized as patient characteristics and device or circuit variables. Relevant patient risk factors include pre-existing comorbidities (e.g., diabetes, chronic cardiorespiratory insufficiency, chronic renal disease), administration of nephrotoxic drugs [e.g., antibiotics, radiocontrast, amphotericin, non-steroidal anti-inflammatory drugs (NSAIDS)]. As already mentioned, AKI is often the result of progression of the baseline disease (e.g., cardiogenic shock, severe heart failure, cardiorenal syndrome) which highlights the importance of timing for MCS. Ideally, MCS should be instituted early enough to avoid further progression to refractory organ failure from hypoperfusion, but late enough to establish reversibility of organ dysfunction prior to exposing the patient to the risks inherent to MCS $(32,33)$. Prolonged requirement for MCS is associated with worse renal outcomes due to increased incidence of sepsis, bleeding and blood transfusions.

\section{VA-ECMO}

Adult patients supported with ECMO who also require dialysis are at significantly greater risk of death than those without renal dysfunction $(4,9)$. Those who require VAECMO following cardiothoracic surgery have substantially higher mortality rates compared with other postcardiothoracic surgical patients, ranging from $31 \%$ to $76 \%$ (1). A national database trial conducted in Taiwan found the requirement for ECMO after cardiac surgery resulted in higher hospital mortality rates $(61.7 \%$ compared with $6.8 \%$ ) (34) compared with a propensitymatched control group that did not require ECMO. The development of dialysis-requiring AKI in such patients portends a rather poor prognosis, with $72 \%$ hospital mortality in the same study (34). Acute renal failure is an independent risk factor for mortality in PCCS patients on ECMO, with odds ratios (OR) between 1.94 and 30.8 depending on the patient group and definition of renal failure $(28,34,35)$.

In a review of 162 ECMO patients (79 with VA-ECMO), those who developed AKI were more likely to have preexisting heart disease, greater severity of disease (SOFA score), higher lactate, increased transfusion and inotropic requirements, and to be treated with VA-ECMO (32). In 200 ECMO patients (89 VA-ECMO), 60\% required renal replacement therapy (RRT) for AKI that was associated with greater severity of disease (SAPS score), higher creatinine on admission and a higher incidence of pre-existing renal dysfunction. The use and duration of RRT was associated with decreased survival at three months (17\%, compared to $53 \%$ in patients without RRT) (9). In 228 consecutive patients treated with VA-ECMO predominantly for PCCS (118, 52\%), the need for RRT was significantly increased in patients with central cannulation (58 of 102 patients, $57 \%$ ) compared to peripheral cannulation (55 of 126 patients, $43 \%$ ). The reduced risk for RRT with peripheral cannulation was also reported in a recent systematic review and meta-analysis (36). Other systematic reviews on VA-ECMO for PCCS found that prolonged preMCS hypotension and poor renal perfusion related to low forward flows on VA-ECMO predisposed to AKI, and the need for RRT was highly associated with mortality $(37,38)$.

In the pediatric ECMO population, recovery of renal function after combination ECMO/RRT therapy occurs frequently $(4,39)$, and has been described in adults with respiratory disease on ECMO (40), however equivalent data in the adult cardiac ECMO population are lacking.

In 43 patients supported with VA-ECMO or continuousflow external VAD for primary graft dysfunction after heart transplantation, the use of VA-ECMO compared favorably 
with VAD in terms of AKI needing RRT [3/27 patients (11\%) vs. $9 / 17$ patients (53\%)] (41).

\section{$V A D$}

The overall issue of LVADs and renal function has recently been reviewed (42). Pre-existing renal dysfunction is seen in about $25 \%$ of patients with advanced heart failure (43) due to decreased cardiac output and kidney perfusion, renal venous congestion and is associated with adverse outcomes and mortality (44-49). This has important implications for candidate selection and ability to bridge to transplant (50). Careful evaluation to determine the cause of renal dysfunction (cardiorenal syndrome or other) is recommended (51). Renal dysfunction appears not to be an absolute contra-indication to LVAD—about $1.5 \%$ of new LVAD patients are receiving dialysis prior to LVAD implantation (52). Patients may experience an improvement in renal function after LVAD implantation due to improved perfusion (53), with some series describing resolution of dialysis requirement shortly after LVAD implantation (54). Recovery of renal function after LVAD placement is associated with improved survival $(50,55)$ however an initial improvement in GFR is often followed by a plateau or slow decline in renal function from around one month to one year post implant $(53,56)$. Postulated reasons include measurement bias with improved muscle mass resulting in higher serum creatinine levels, devicerelated hemolysis, right ventricular failure and non-pulsatile flow (52). Development of AKI in the immediate post implantation period is associated with high short-term mortality risk. A retrospective study of 389 LVAD patients, 44 of whom required post implantation RRT, found a $73.9 \%$ hospital mortality for patients requiring de novo RRT in the first month post implantation, and a significantly greater duration of hospital admission (57). In a review of 520 patients following LVAD implantation for advanced, refractory chronic heart failure, 75 (14\%) developed AKI that was an independent predictor of mortality (hazard ratio 1.54). Pre-operative MCS and prolonged cardiopulmonary bypass time were independent predictors of AKI (47). An observational study of 100 LVAD patients reported that the $28 \%$ who developed post-operative acute renal failure were more likely to have been intubated pre-operatively and had higher pre-operative central venous pressure (46). In 389 patients undergoing LVAD implantation, risk factors for requirement of RRT (44 patients, 12\%) were pre-implantation renal dysfunction (estimated glomerular filtration rate $<40 \mathrm{~mL} \cdot \mathrm{min}^{-1} \cdot \mathrm{m}^{-2}$ ), proteinuria and low hemoglobin (57). Diabetes mellitus was the only significant predictor for AKI (44 patients, 28\%) in another study of 157 LVAD recipients (58). An analysis of $>12,000$ patients following LVAD implantation in the Interagency Registry for Mechanically Assisted Circulatory Support (INTERMACS) database showed that $12.3 \%$ LVAD patients developed AKI (59) although second and third generation (continuous flow) LVADs are associated with a reduced incidence of renal failure compared with the first generation (pulsatile flow) devices $(60,61)$.

\section{Management}

\section{General management and prevention}

Prevention and treatment of perioperative renal dysfunction in cardiothoracic surgical patients begins in the preoperative period and continues throughout the hospital admission. Management of at-risk patients includes avoidance of nephrotoxins, optimization of metabolic and intravascular volume status and prophylaxis against thromboembolism.

Protocols aimed at protecting the kidneys from the nephrotoxic effects of radiocontrast are especially important if cardiothoracic surgery follows rapidly after coronary angiography or contrast CT scanning. Treatment with $\mathrm{N}$-Acetyl Cysteine and sodium bicarbonate are not supported by strong evidence, but ensuring adequate perfusion with use of iso-osmolar crystalloid infusion and using low-osmolarity contrast agents at the lowest possible dose is recommended (62). Metformin is usually withheld around the time of coronary angiography and peri-operatively, and caution should be exercised in the administration of agents used in management of heart failure such as ACE-inhibitors and angiotensin receptor blockers, which may be associated with renal injury in susceptible individuals (63). The use of NSAIDs for postoperative analgesia is not recommended in patients with renal dysfunction. Many immunosuppressant drugs used in transplant patients have important nephrotoxic effects and require careful selection and dose adjustment.

Meticulous attention should be paid to blood glucose management, as $\mathrm{HbA1C}$ elevation pre-operatively is associated with post-operative complications. Adequate blood sugar control in the intra- and post-operative period, including the use of insulin infusions where required, is associated with a lower rate of renal injury (64). 
Embolic phenomena encountered in the cardiothoracic surgical setting include thromboembolic complications, as well as embolism of atheroma and other intra-cardiac material such as valvular vegetations, all of which may occlude the renal circulation causing renal dysfunction. Prevention includes careful pre-, intra-, and postoperative evaluation for intracardiac sources of embolism, especially for patients at increased risk. Echocardiographic examination is warranted in patients with persistent or recurrent atrial fibrillation, severe LV systolic dysfunction especially with LV aneurysm, or clinical suspicion of endocarditis. Transesophageal echocardiogram may be required for complete examination and is recommended in all patients undergoing open-heart surgery $(65,66)$. Patients on mechanical support are anticoagulated prophylactically in the absence of contraindications. Any suspicion of device thrombus formation should prompt further evaluation using appropriate imaging, titration of anticoagulation therapy and consideration of thrombolysis or device replacement.

\section{Hemodynamic support}

The goal of hemodynamic support with respect to renal protection is to enhance renal perfusion by optimizing volume status, cardiac output and blood pressure while avoiding fluid overload and venous congestion. This applies to patients with pre-operative cardiogenic shock, those on cardiopulmonary bypass or requiring circulatory support during the operative phase, and those with cardiovascular instability in the post-operative period.

Because duration of CPB is associated with renal injury, bypass time should be minimized or avoided by the use of off-pump techniques. Management of the CPB circuit with regards to flow and hemodilution represent opportunities for renal protection but are outside the scope of this review.

Practitioners must be vigilant for post-operative complications that may impair end-organ perfusion such as bleeding, pericardial tamponade, myocardial stunning associated with inadequate cardiac protection, coronary graft occlusion, dysfunction of replaced or repaired heart valves, or heart or lung allograft dysfunction.

A combination of clinical, echocardiographic and invasive (pulmonary artery catheter or other transpulmonary thermodilution catheters) monitoring may be used to guide hemodynamic management including volume resuscitation (or removal), vasopressor (or vasodilator) and inotrope therapy.

Where non-invasive supports are insufficient, the timely institution of MCS should be pursued before established end-organ dysfunction ensues. Thereafter, manipulation of renal perfusion requires a detailed understanding of interactions between the patient's physiology and the MCS device.

Ensuring adequate systemic perfusion requires a detailed understanding of patient-device interactions and careful balance between device function and native cardiac output. This is particularly important when utilizing peripheral VA ECMO, when high flows increase LV afterload and may prevent opening of the aortic valve. Titration of VAD pump speed to optimal flow and pulsatility index should be carried out in collaboration with a specialist VAD team. The ICU and surgical team must also be prepared for early recognition and treatment of device-related complications that may have deleterious effects on systemic perfusion including malpositioning, thrombosis, bleeding, and preload or afterload issues affecting device function.

\section{Volume management}

In the initial resuscitation and management of shocked patients, administration of intravenous fluids to augment cardiac output and organ perfusion is almost universal, and forms an important component of renal protection. Adequate volume resuscitation and early recognition and management of bleeding are essential in the early resuscitative phase and in the immediate post-cardiothoracic surgical period.

For patients on mechanical support, blood flow pumps whether centrifugal or axial, are pre-load dependent. Adequate fluid resuscitation is vital in both ECMO and VAD patients to prevent "suck-down" or "access insufficiency" events that impair device function and can cause severe hemodynamic instability with resulting impairment in renal perfusion.

Conversely however, there is increasing recognition that after initial volume resuscitation and in the absence of ongoing losses, patients do not remain volume deplete but often tend towards fluid overload. Positive fluid balance is associated with significant deleterious effects in the critically ill (67). The association between fluid overload and outcome is multifactorial and includes poor wound healing due to subcutaneous edema, difficulty weaning from mechanical ventilation due to cardiogenic and non-cardiogenic pulmonary edema, exacerbation of critical illness-associated weakness and immobility due to increased body mass, and end-organ dysfunction due to right ventricular volume 
overload, right ventricular failure and venous congestion. The causative association of renal dysfunction and volume overload is bidirectional, with oliguria and progressive fluid overload resulting in right ventricular dysfunction and venous congestion, which in turn exacerbates renal dysfunction.

Fluid overload is associated with increased mortality in patients supported by ECMO $(39,68,69)$. Observational data in adult patients requiring ECMO (68) have demonstrated a strong association between positive fluid balance at day 3 and increase in 90-day mortality. The Extracorporeal Life Support Organization (ELSO) suggests the fluid management goal in ECMO patients is to return the extracellular fluid volume to normal (dry weight) and maintain it there (70).

Management of volume status in LVAD-supported patients is particularly important as it relates to right ventricular dysfunction, especially in the early post-operative period where right ventricular preload is rapidly increased due to augmentation of left ventricular output by the LVAD. In 100 LVAD patients, 28 of whom developed postoperative ARF, higher pre-operative CVP was independently associated with post-operative acute renal failure postoperatively suggesting an association between renal failure and subclinical right ventricular dysfunction (46). In another observational study of 520 patients receiving axial flow (HeartMate II) or centrifugal flow (HVAD) devices, an association between post-operative AKI and right heart failure was noted (47). Careful support of right heart function may help to preserve renal function and therefore treatments should be directed at avoiding hypoxic pulmonary vasoconstriction, high airway pressures, and respiratory acidosis, consideration of pulmonary vasodilators, use of appropriate inotropic agents including milrinone, and consideration of Right Ventricular Assist Device implantation or temporary right heart support with Ventricular-Pulmonary Arterial ECMO, where required.

\section{Dialysis}

Dialysis is required where diuretic therapy has failed to control volume status, or in cases of severe biochemical derangement. An overview of renal replacement techniques is provided in Table 1. Timing (early or late), specific indications, mode, and delivery (including vascular access) vary depending on local practice and clinical context. Potential advantages of dialysis in the post-operative MCS patient include:

Careful control of fluid balance: dialysis provides more predictable fluid removal than diuretic therapy, avoids potential side effects of high dose diuretics such as ototoxicity (71) and allows enhanced nutritional support (4); correction of metabolic acidosis, electrolyte disturbances and uremia; removal of proinflammatory cytokines and other inflammatory mediators, which theoretically may improve hemodynamic status (72); reduction in the metabolic demands on the kidney which could improve renal recovery (26).

Disadvantages of dialysis include: added complexity and cost; risk of complications due to requirement for dialysis catheter insertion or accessing of the ECMO circuit for hemofiltration; complications related to the haemodialysis circuit including increased shear stress, wall impact forces and exposure to non-endotheliazed surfaces causing hemolysis, fibrinolysis and inflammatory activation $(4,71)$; intolerance of hemodialysis, particularly at time of commencement, due to either hemodynamic instability or flow- or pressure-related device dysfunction. Suction events or access insufficiency may occur in both ECMO and VAD patients, if high dialysis flow rates or rapid fluid removal is attempted.

Both ECMO and LVAD patients treated with hemodialysis display higher mortality rates than those who do not require dialysis however this observation is likely related to severity of illness rather than the treatment itself (5). No randomized controlled trials have examined the provision of dialysis in these patient populations with regards to timing, indications or mode. A cross-sectional survey of 65 ECMO centers (73), 40\% of which treated adult patients, found the most common indication for dialysis was treatment (43\%) or prevention (16\%) of fluid overload. Other indications reported in observational studies of patients on ECMO have included metabolic acidosis, electrolyte disturbances (especially hyperkalemia) and elevated blood urea levels (4,32).

An attractive notion that hemodialysis may improve hemodynamic stability in shocked patients, by correcting biochemical derangements and removing inflammatory mediators, has not been confirmed by definitive evidence. The HEROICS study of high volume hemofiltration in PCCS patients failed to show a mortality benefit in the intervention arm, although HVHF patients were more rapidly weaned off catecholamines (72).

\section{Provision of dialysis in ECMO patients}

Most patients on ECMO who require dialysis are treated 
Table 1 Methods for combining renal replacement therapy with veno-arterial extracorporeal membrane oxygenation

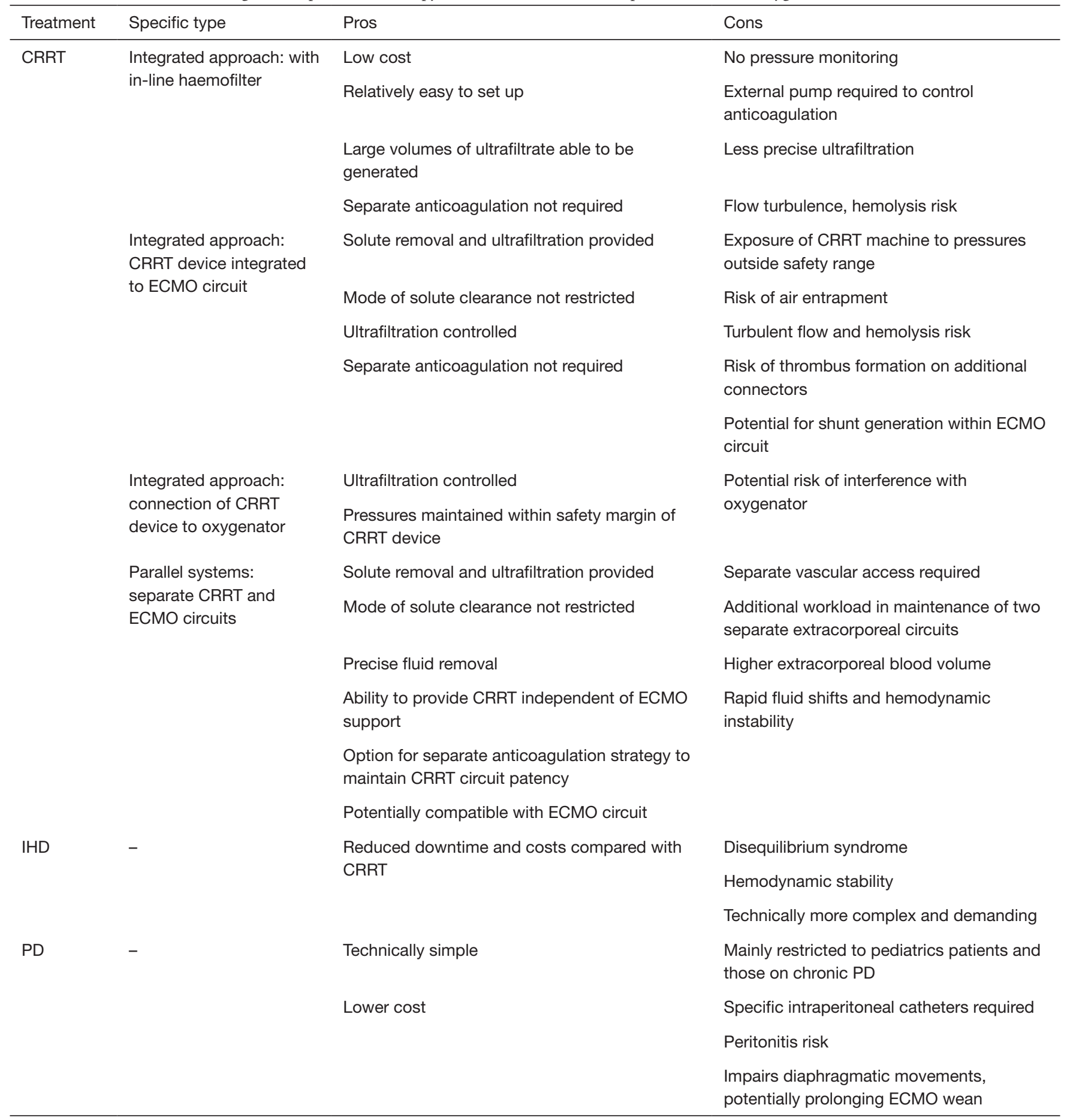

CRRT, continuous renal replacement therapy; ECMO, extracorporeal membrane oxygenation; IHD, intermittent hemodialysis; PD, peritoneal dialysis. 


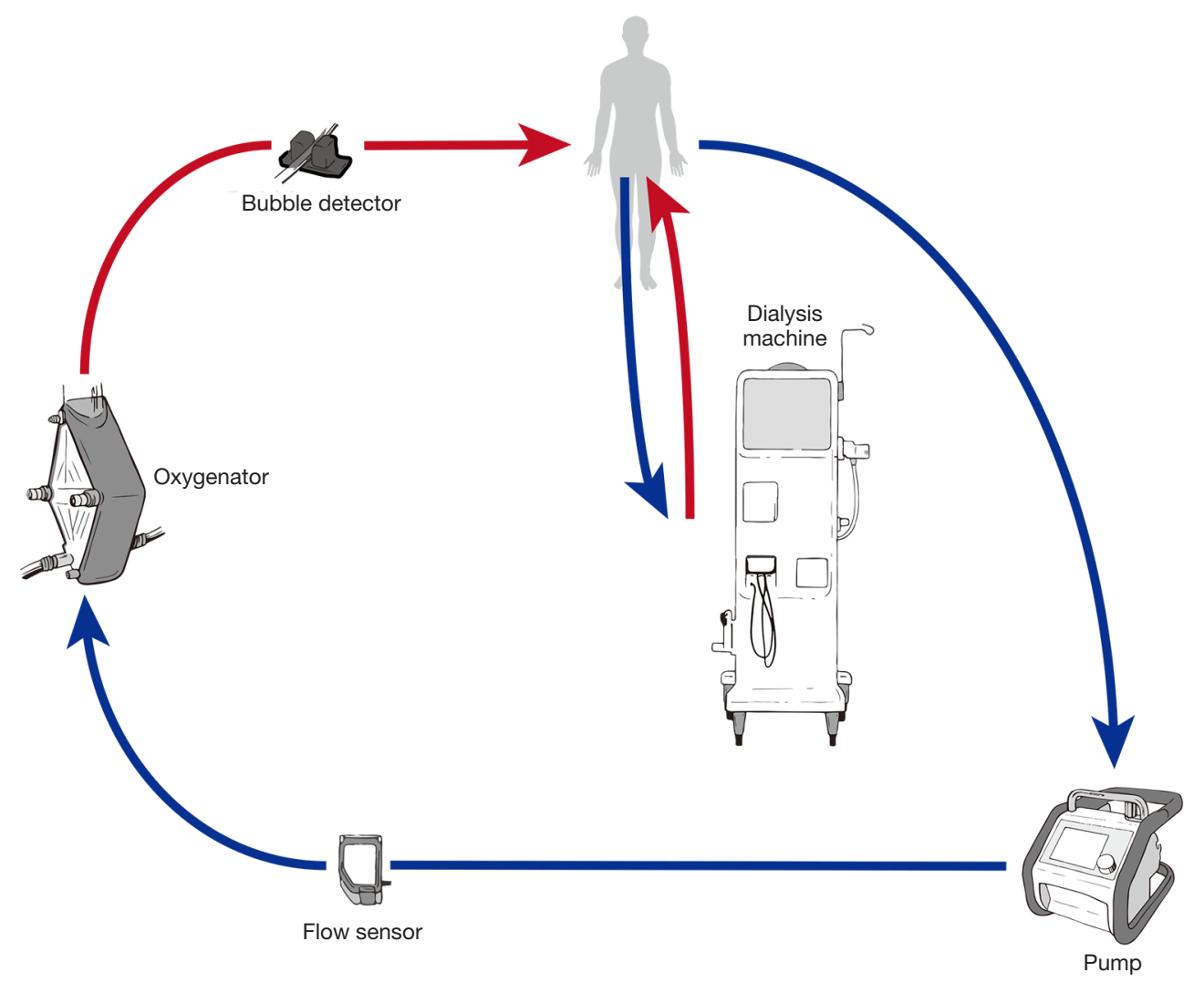

Figure 2 Independent access for providing renal replacement therapy in patients following implantation of mechanical circulatory support.

with continuous renal replacement therapy (CRRT) as this is associated with less hemodynamic disturbance than the higher flows and faster ultrafiltration (UF) required with intermittent hemodialysis $(33,71)$. Peritoneal dialysis is well described in neonates and pediatric patients on ECMO but has not been widely used in adults.

When applying CRRT to patients on ECMO support, there are three options for connecting the CRRT device to the patient: Independent CRRT access using a vascular catheter, introduction of an inline hemofilter into the ECMO circuit, and introduction of a CRRT device into the ECMO circuit. Important aspects, advantages and disadvantages of each are described below (see also Table 1).

\section{Independent CRRT access (Figure 2)}

Using a separate vascular access catheter for dialysis reduces ECMO-CRRT circuit interactions and eliminates the requirement to access the ECMO circuit to connect or change the CRRT filter. Procedural risks of inserting the dialysis vascular access catheter include bleeding, damage to vascular and cardiac structures and pneumothorax. Other risks include catheter site infection or central line associated blood stream infection (CLABSI) and the potential for poor dialysis flows due to catheter misplacement, occlusion or thrombosis. Options for circuit anticoagulation include systemic anticoagulation, which may already be in use for the ECMO circuit. If the perceived bleeding risk is high, regional anticoagulation of the CRRT device is possible using prefilter citrate with calcium replacement.

\section{Introduction of an inline hemofiltration device into the ECMO circuit (Figure 3)}

This simple, relatively inexpensive technique is in common usage (4) and utilizes infusion pumps to provide predominantly ultrafiltration with limited solute clearance (71). Disadvantages include the lack of pressure monitors and alarms in the hemofilter device and the use of infusion pumps to measure fluid removal, which may result in inaccurate measurement and prevents fine control over ultrafiltration. 


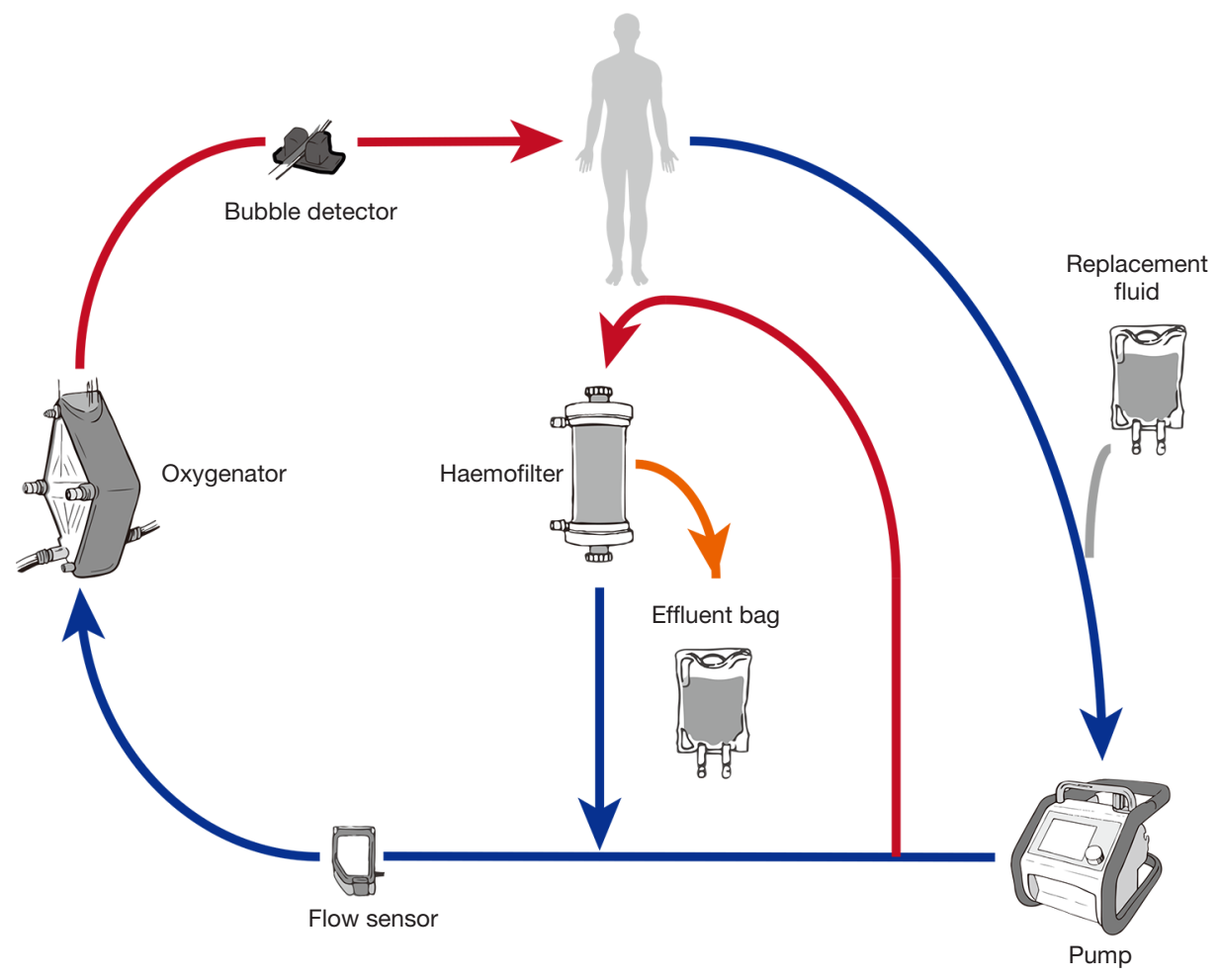

Figure 3 In-line hemofiltration to provide renal replacement therapy in patients following implantation of mechanical circulatory support.

\section{Introduction of a CRRT device into the ECMO circuit (Figure 4)}

This is probably the most common method for dialysis in patients on ECMO (73). It allows precise control of fluid management (4) and possibly longer filter life than independent CRRT access (74). Safe delivery requires a good understanding of pump mechanics and intra-circuit pressures. For use with ECMO circuits containing a centrifugal pump, connecting the CRRT device in the postpump (positive pressure) section theoretically reduces the risk of air entrainment into the circuit. Returning the blood from the CRRT device to the pre-oxygenator segment allows the ECMO membrane to act as a trap for air bubbles and clots inadvertently returned from the CRRT device (Figure 4, solid connecting lines to dialysis machine). This represents off-license use of CRRT machines, most of which are manufactured to tolerate only small positive pressures $(0-20 \mathrm{mmHg})$ and therefore high pressure alarms on the CRRT device can be problematic especially at high ECMO flow rates (71). This can be offset by returning the blood or connecting the entire circuit to the pre-pump (negative pressure) segment of the ECMO circuit (75) however this may increase the risk of air embolism. It is also possible to connect using existing Luer lock connectors on the inlet and outlet ports of the oxygenator (Figure 4, dashed lines to dialysis machine) (76).

Any integrated system results in a shunt within the ECMO circuit, which theoretically could affect the oxygenation and disturb blood flows. The shunt fraction is usually not above $5 \%$ (i.e., $200 \mathrm{~mL} / \mathrm{min}$ from $4,000 \mathrm{~mL} / \mathrm{min}$ ECMO flow) (71).

\section{Provision of dialysis in VAD patients}

Patients who require RRT for AKI post VAD insertion are generally treated with CRRT by insertion of a dialysis catheter, in the short term. Careful hemodynamic monitoring is of vital importance to prevent suction events associated with ultrafiltration, and in the intensive care unit is accomplished using standard monitoring for postoperative VAD patients. A low pulsatility index may be suggestive of excessive fluid removal $(42,51)$.

For patients in whom renal failure does not resolve and who require long-term dialysis, intermittent hemodialysis may be achieved using a long-term (tunneled) vascular access catheter. This carries a risk of CLABSI, which 


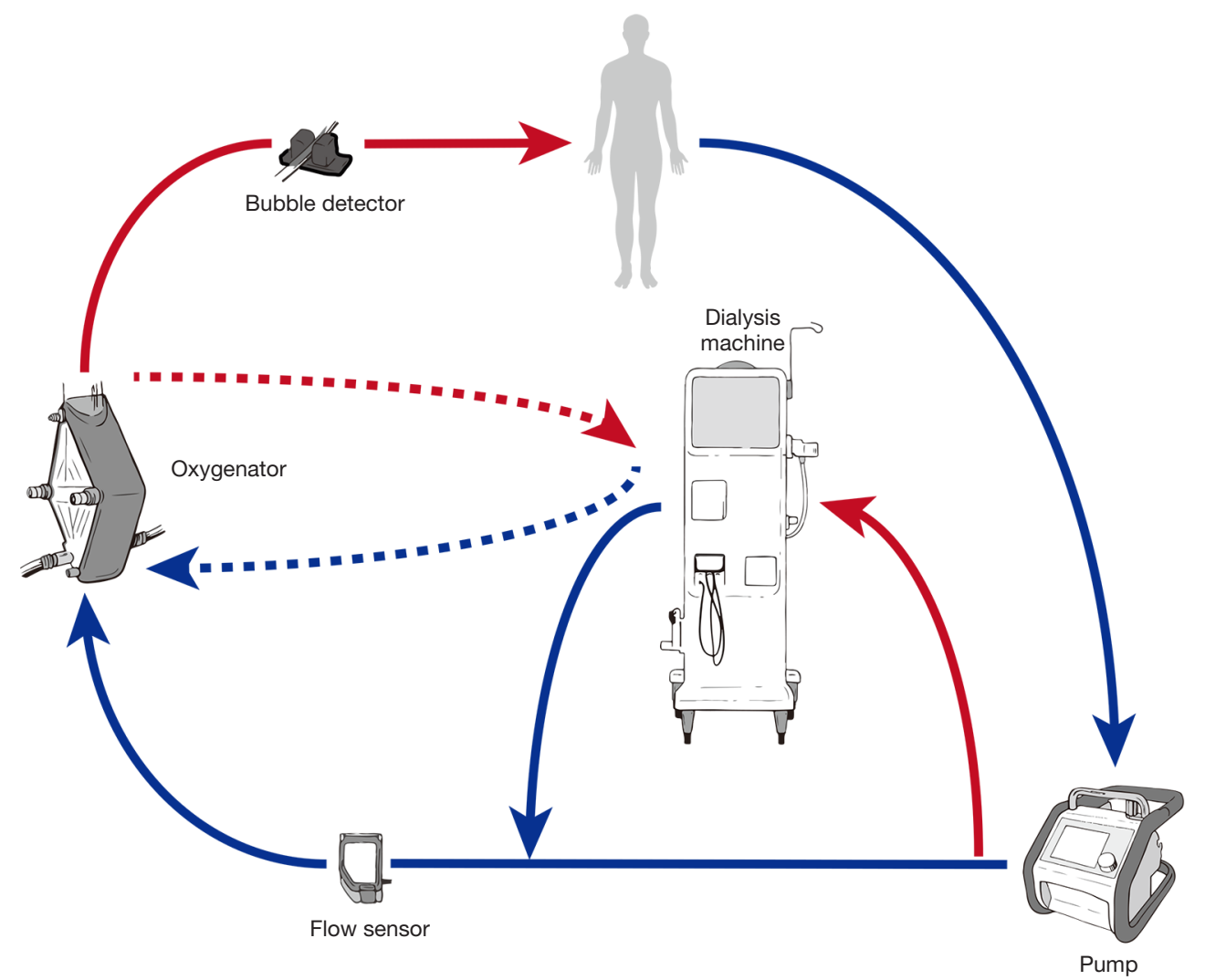

Figure 4 Introduction of renal replacement therapy into the circuit following implantation of mechanical circulatory support.

may be disastrous if organisms are seeded to the VAD. Formation of arteriovenous fistulae in such patients is therefore recommended by some authors (51), although maturation of the fistula is potentially problematic due to the lack of pulsatile blood flow and may require balloonassisted maturation (42). Hemodynamic monitoring during intermittent dialysis sessions becomes more problematic due to lack of pulsatility rendering standard non-invasive blood pressure measurements inaccurate, and close collaboration between the VAD team and the dialysis center is required (51).

Peritoneal dialysis is frequently used in heart failure patients (42) and has the advantages of gentle fluid and solute shift with improved hemodynamic tolerability. There are published case studies of using peritoneal dialysis in VAD patients $(77,78)$, where the lower risk of infection in peritoneal dialysis compared with hemodialysis makes for an attractive option. The risk of infection to the VAD driveline is a substantial concern however, and peritoneal dialysis is not possible with sub-diaphragmatic devices.

\section{Conclusions}

$\mathrm{AKI}$ is common during ECMO support and is associated with increased mortality. In patients receiving durable VAD support, some degree of renal dysfunction is common in the pre-operative phase as a result of chronic heart failure, however the development of new renal failure following VAD implantation appears lower compared with ECMO. The complex interplay between patient factors including pre-morbid risks, pre- and intra-MCS treatment factors remains incompletely investigated and understood. Management options begin in the pre-operative phase and continue throughout the post-operative period, with particular importance placed on hemodynamic management and fluid balance. Dialysis in the early post-operative phase is most commonly provided using continuous renal replacement therapy. Configuration and delivery options for renal replacement therapy depend on patient factors, device factors, and institutional experience. 


\section{Acknowledgments}

The authors are grateful for the artwork provided by Johanna Aneman (www.darkhoursco.com).

Funding: None.

\section{Footnote}

Provenance and Peer Review: This article was commissioned by the Guest Editor (Kamen Valchanov) for the series "Perioperative Management of Patients with undergoing Mechanical Circulatory Support" published in Annals of Translational Medicine. The article was sent for external peer review organized by the Guest Editor and the editorial office.

Conflicts of Interest: All authors have completed the ICMJE uniform disclosure form. (available at http://dx.doi. org/10.21037/atm-20-1172). The series "Perioperative Management of Patients with undergoing Mechanical Circulatory Support" was commissioned by the editorial office without any funding or sponsorship. The authors have no other conflicts of interest to declare.

Ethical Statement: The authors are accountable for all aspects of the work in ensuring that questions related to the accuracy or integrity of any part of the work are appropriately investigated and resolved.

Open Access Statement: This is an Open Access article distributed in accordance with the Creative Commons Attribution-NonCommercial-NoDerivs 4.0 International License (CC BY-NC-ND 4.0), which permits the noncommercial replication and distribution of the article with the strict proviso that no changes or edits are made and the original work is properly cited (including links to both the formal publication through the relevant DOI and the license). See: https://creativecommons.org/licenses/by-nc-nd/4.0/.

\section{References}

1. Lorusso R, Raffa GM, Kowalewski M, et al. Structured review of post-cardiotomy extracorporeal membrane oxygenation: Part 2-pediatric patients. J Heart Lung Transplant 2019;38:1144-61.

2. Whitman GJ. Extracorporeal membrane oxygenation for the treatment of postcardiotomy shock. J Thorac Cardiovasc Surg 2017;153:95-101.
3. Wang L, Wang H, Hou X. Clinical Outcomes of Adult Patients Who Receive Extracorporeal Membrane Oxygenation for Postcardiotomy Cardiogenic Shock: A Systematic Review and Meta-Analysis. J Cardiothorac Vasc Anesth 2018;32:2087-93.

4. Chen H, Yu RG, Yin NN, et al. Combination of extracorporeal membrane oxygenation and continuous renal replacement therapy in critically ill patients: a systematic review. Crit Care 2014;18:675.

5. Han SS, Kim HJ, Lee SJ, et al. Effects of Renal Replacement Therapy in Patients Receiving Extracorporeal Membrane Oxygenation: A Meta-Analysis. Ann Thorac Surg 2015;100:1485-95.

6. Lin CY, Tsai FC, Tian YC, et al. Evaluation of outcome scoring systems for patients on extracorporeal membrane oxygenation. Ann Thorac Surg 2007;84:1256-62.

7. Chen YC, Tsai FC, Chang CH, et al. Prognosis of patients on extracorporeal membrane oxygenation: the impact of acute kidney injury on mortality. Ann Thorac Surg 2011;91:137-42.

8. Lin CY, Chen YC, Tsai FC, et al. RIFLE classification is predictive of short-term prognosis in critically ill patients with acute renal failure supported by extracorporeal membrane oxygenation. Nephrol Dial Transplant 2006;21:2867-73.

9. Kielstein JT, Heiden AM, Beutel G, et al. Renal function and survival in 200 patients undergoing ECMO therapy. Nephrol Dial Transplant 2013;28:86-90.

10. Yalcin YC, Bunge JJH, Guven G, et al. Acute kidney injury following left ventricular assist device implantation: Contemporary insights and future perspectives. J Heart Lung Transplant 2019;38:797-805.

11. Muslem R, Caliskan K, Akin S, et al. Acute kidney injury and 1-year mortality after left ventricular assist device implantation. J Heart Lung Transplant 2018;37:116-23.

12. Evans RG, Ince C, Joles JA, et al. Haemodynamic influences on kidney oxygenation: clinical implications of integrative physiology. Clin Exp Pharmacol Physiol 2013;40:106-22.

13. Kilburn DJ, Shekar K, Fraser JF. The Complex Relationship of Extracorporeal Membrane Oxygenation and Acute Kidney Injury: Causation or Association? Biomed Res Int 2016;2016:1094296.

14. Bellomo R, Kellum JA, Ronco C. Acute kidney injury. Lancet 2012;380:756-66.

15. Prowle J, Bagshaw SM, Bellomo R. Renal blood flow, fractional excretion of sodium and acute kidney injury: time for a new paradigm? Curr Opin Crit Care 
2012;18:585-92.

16. Matejovic M, Ince C, Chawla LS, et al. Renal Hemodynamics in AKI: In Search of New Treatment Targets. J Am Soc Nephrol 2016;27:49-58.

17. Legrand M, Mik EG, Johannes T, et al. Renal hypoxia and dysoxia after reperfusion of the ischemic kidney. Mol Med 2008;14:502-16.

18. Salmon AH, Ferguson JK, Burford JL, et al. Loss of the endothelial glycocalyx links albuminuria and vascular dysfunction. J Am Soc Nephrol 2012;23:1339-50.

19. Sharfuddin AA, Molitoris BA. Pathophysiology of ischemic acute kidney injury. Nat Rev Nephrol 2011;7:189-200.

20. Ince C. The central role of renal microcirculatory dysfunction in the pathogenesis of acute kidney injury. Nephron Clin Pract 2014;127:124-8.

21. Kumar AB, Suneja M. Cardiopulmonary bypass-associated acute kidney injury. Anesthesiology 2011;114:964-70.

22. Freeland K, Hamidian Jahromi A, Duvall LM, et al. Postoperative blood transfusion is an independent predictor of acute kidney injury in cardiac surgery patients. J Nephropathol 2015;4:121-6.

23. de Waal EEC, van Zaane B, van der Schoot MM, et al. Vasoplegia after implantation of a continuous flow left ventricular assist device: incidence, outcomes and predictors. BMC Anesthesiol 2018;18:185.

24. Moore JP, Dyson A, Singer M, et al. Microcirculatory dysfunction and resuscitation: why, when, and how. Br J Anaesth 2015;115:366-75.

25 . Ince $C$. The rationale for microcirculatory guided fluid therapy. Curr Opin Crit Care 2014;20:301-8.

26. Villa G, Katz N, Ronco C. Extracorporeal Membrane Oxygenation and the Kidney. Cardiorenal Med 2015;6:50-60.

27. Khwaja A. KDIGO clinical practice guidelines for acute kidney injury. Nephron Clin Pract 2012;120:c179-84.

28. Yan X, Jia S, Meng X, et al. Acute kidney injury in adult postcardiotomy patients with extracorporeal membrane oxygenation: evaluation of the RIFLE classification and the Acute Kidney Injury Network criteria. Eur J Cardiothorac Surg 2010;37:334-8.

29. Tsai TY, Chien H, Tsai FC, et al. Comparison of RIFLE, AKIN, and KDIGO classifications for assessing prognosis of patients on extracorporeal membrane oxygenation. J Formos Med Assoc 2017;116:844-51.

30. Englberger L, Suri RM, Li Z, et al. Clinical accuracy of RIFLE and Acute Kidney Injury Network (AKIN) criteria for acute kidney injury in patients undergoing cardiac surgery. Crit Care 2011;15:R16.
31. Srisawat N, Kellum JA. The Role of Biomarkers in Acute Kidney Injury. Crit Care Clin 2020;36:125-40.

32. Antonucci E, Lamanna I, Fagnoul D, et al. The Impact of Renal Failure and Renal Replacement Therapy on Outcome During Extracorporeal Membrane Oxygenation Therapy. Artif Organs 2016;40:746-54.

33. Askenazi DJ, Selewski DT, Paden ML, et al. Renal replacement therapy in critically ill patients receiving extracorporeal membrane oxygenation. Clin J Am Soc Nephrol 2012;7:1328-36.

34. Chen SW, Tsai FC, Lin YS, et al. Long-term outcomes of extracorporeal membrane oxygenation support for postcardiotomy shock. J Thorac Cardiovasc Surg 2017;154:469-77.e2.

35. Rastan AJ, Dege A, Mohr M, et al. Early and late outcomes of 517 consecutive adult patients treated with extracorporeal membrane oxygenation for refractory postcardiotomy cardiogenic shock. J Thorac Cardiovasc Surg 2010;139:302-11, 11.e1.

36. Raffa GM, Kowalewski M, Brodie D, et al. Meta-Analysis of Peripheral or Central Extracorporeal Membrane Oxygenation in Postcardiotomy and Non-Postcardiotomy Shock. Ann Thorac Surg 2019;107:311-21.

37. Khorsandi M, Dougherty S, Bouamra O, et al. Extracorporeal membrane oxygenation for refractory cardiogenic shock after adult cardiac surgery: a systematic review and meta-analysis. J Cardiothorac Surg 2017;12:55.

38. Fukuhara S, Takeda K, Garan AR, et al. Contemporary mechanical circulatory support therapy for postcardiotomy shock. Gen Thorac Cardiovasc Surg 2016;64:183-91.

39. Paden ML, Warshaw BL, Heard ML, et al. Recovery of renal function and survival after continuous renal replacement therapy during extracorporeal membrane oxygenation. Pediatr Crit Care Med 2011;12:153-8.

40. McCanny P, Smith MW, O'Brien SG, et al. Fluid Balance and Recovery of Native Lung Function in Adult Patients Supported by Venovenous Extracorporeal Membrane Oxygenation and Continuous Renal Replacement Therapy. ASAIO J 2019;65:614-9.

41. Takeda K, Li B, Garan AR, et al. Improved outcomes from extracorporeal membrane oxygenation versus ventricular assist device temporary support of primary graft dysfunction in heart transplant. J Heart Lung Transplant 2017;36:650-6.

42. Kamboj M, Kazory A. Left Ventricular Assist Device and the Kidney: Getting to the Heart of the Matter. Blood Purif 2019;48:289-98.

43. Hebert K, Dias A, Delgado MC, et al. Epidemiology 
and survival of the five stages of chronic kidney disease in a systolic heart failure population. Eur J Heart Fail 2010;12:861-5.

44. Cowger J, Sundareswaran K, Rogers JG, et al. Predicting survival in patients receiving continuous flow left ventricular assist devices: the HeartMate II risk score. J Am Coll Cardiol 2013;61:313-21.

45. Kirklin JK, Naftel DC, Kormos RL, et al. Quantifying the effect of cardiorenal syndrome on mortality after left ventricular assist device implant. J Heart Lung Transplant 2013;32:1205-13.

46. Borgi J, Tsiouris A, Hodari A, et al. Significance of postoperative acute renal failure after continuous-flow left ventricular assist device implantation. Ann Thorac Surg 2013;95:163-9.

47. Anjum A, Kurihara C, Critsinelis A, et al. Acute kidney injury after implantation of a left ventricular assist device: a comparison of axial-flow (HeartMate II) and centrifugalflow (HeartWare HVAD) devices. J Artif Organs 2018;21:285-92.

48. Moller-Helgestad OK, Poulsen CB, Christiansen $\mathrm{EH}$, et al. Support with intra-aortic balloon pump vs. Impella2.5(R) and blood flow to the heart, brain and kidneys - an experimental porcine model of ischaemic heart failure. Int J Cardiol 2015;178:153-8.

49. Kirklin JK, Pagani FD, Kormos RL, et al. Eighth annual INTERMACS report: Special focus on framing the impact of adverse events. J Heart Lung Transplant 2017;36:1080-6.

50. Sandner SE, Zimpfer D, Zrunek P, et al. Renal function and outcome after continuous flow left ventricular assist device implantation. Ann Thorac Surg 2009;87:1072-8.

51. Patel AM, Adeseun GA, Ahmed I, et al. Renal failure in patients with left ventricular assist devices. Clin J Am Soc Nephrol 2013;8:484-96.

52. Tromp TR, de Jonge N, Joles JA. Left ventricular assist devices: a kidney's perspective. Heart Fail Rev 2015;20:519-32.

53. Hasin T, Topilsky Y, Schirger JA, et al. Changes in renal function after implantation of continuousflow left ventricular assist devices. J Am Coll Cardiol 2012;59:26-36.

54. Schmack B, Grossekettler L, Weymann A, et al. Prognostic relevance of hemodialysis for short-term survival in patients after LVAD implantation. Sci Rep 2018;8:8546.

55. Butler J, Geisberg C, Howser R, et al. Relationship between renal function and left ventricular assist device use. Ann Thorac Surg 2006;81:1745-51.
56. Brisco MA, Kimmel SE, Coca SG, et al. Prevalence and prognostic importance of changes in renal function after mechanical circulatory support. Circ Heart Fail 2014;7:68-75.

57. Topkara VK, Coromilas EJ, Garan AR, et al. Preoperative Proteinuria and Reduced Glomerular Filtration Rate Predicts Renal Replacement Therapy in Patients Supported With Continuous-Flow Left Ventricular Assist Devices. Circ Heart Fail 2016;9.

58. Naik A, Akhter SA, Fedson S, et al. Acute kidney injury and mortality following ventricular assist device implantation. Am J Nephrol 2014;39:195-203.

59. Kirklin JK, Naftel DC, Pagani FD, et al. Seventh INTERMACS annual report: 15,000 patients and counting. J Heart Lung Transplant 2015;34:1495-504.

60. Slaughter MS, Rogers JG, Milano CA, et al. Advanced heart failure treated with continuous-flow left ventricular assist device. N Engl J Med 2009;361:2241-51.

61. Aaronson KD, Slaughter MS, Miller LW, et al. Use of an intrapericardial, continuous-flow, centrifugal pump in patients awaiting heart transplantation. Circulation 2012;125:3191-200.

62. Patschan D, Buschmann I, Ritter O. Contrast-Induced Nephropathy: Update on the Use of Crystalloids and Pharmacological Measures. Int J Nephrol 2018;2018:5727309.

63. Bellomo R, Auriemma S, Fabbri A, et al. The pathophysiology of cardiac surgery-associated acute kidney injury (CSA-AKI). Int J Artif Organs 2008;31:166-78.

64. Lazar HL, McDonnell M, Chipkin SR, et al. The Society of Thoracic Surgeons practice guideline series: Blood glucose management during adult cardiac surgery. Ann Thorac Surg 2009;87:663-9.

65. Saric M, Armour AC, Arnaout MS, et al. Guidelines for the Use of Echocardiography in the Evaluation of a Cardiac Source of Embolism. J Am Soc Echocardiogr 2016;29:1-42.

66. American Society of A, Society of Cardiovascular Anesthesiologists Task Force on Transesophageal E. Practice guidelines for perioperative transesophageal echocardiography. An updated report by the American Society of Anesthesiologists and the Society of Cardiovascular Anesthesiologists Task Force on Transesophageal Echocardiography. Anesthesiology 2010;112:1084-96.

67. National Heart, Lung, and Blood Institute Acute Respiratory Distress Syndrome (ARDS) Clinical Trials Network; Herbert P Wiedemann, Wheeler AP, et al. 
Comparison of two fluid-management strategies in acute lung injury. N Engl J Med 2006;354:2564-75.

68. Schmidt M, Bailey M, Kelly J, et al. Impact of fluid balance on outcome of adult patients treated with extracorporeal membrane oxygenation. Intensive Care Med 2014;40:1256-66.

69. Hoover NG, Heard M, Reid C, et al. Enhanced fluid management with continuous venovenous hemofiltration in pediatric respiratory failure patients receiving extracorporeal membrane oxygenation support. Intensive Care Med 2008;34:2241-7.

70. Extracorporeal Life Support: The ELSO Red Book. 5th ed. Ann Arbor, Michigan, USA: Extracorporeal Life Support Organization, 2017.

71. Ostermann M, Connor M, Jr., Kashani K. Continuous renal replacement therapy during extracorporeal membrane oxygenation: why, when and how? Curr Opin Crit Care 2018;24:493-503.

72. Combes A, Brechot N, Amour J, et al. Early High-Volume Hemofiltration versus Standard Care for Post-Cardiac Surgery Shock. The HEROICS Study. Am J Respir Crit Care Med 2015;192:1179-90.

73. Fleming GM, Askenazi DJ, Bridges BC, et al. A

Cite this article as: Austin D, McCanny P, Aneman A. Post-operative renal failure management in mechanical circulatory support patients. Ann Transl Med 2020;8(13):833. doi: 10.21037/atm-20-1172 multicenter international survey of renal supportive therapy during ECMO: the Kidney Intervention During Extracorporeal Membrane Oxygenation (KIDMO) group. ASAIO J 2012;58:407-14.

74. Santiago MJ, Sanchez A, Lopez-Herce J, et al. The use of continuous renal replacement therapy in series with extracorporeal membrane oxygenation. Kidney Int 2009;76:1289-92.

75. de Tymowski C, Desmard M, Lortat-Jacob B, et al. Impact of connecting continuous renal replacement therapy to the extracorporeal membrane oxygenation circuit. Anaesth Crit Care Pain Med 2018;37:557-64.

76. Seczynska B, Krolikowski W, Nowak I, et al. Continuous renal replacement therapy during extracorporeal membrane oxygenation in patients treated in medical intensive care unit: technical considerations. Ther Apher Dial 2014;18:523-34.

77. Guglielmi AA, Guglielmi KE, Bhat G, et al. Peritoneal dialysis after left ventricular assist device placement. ASAIO J 2014;60:127-8.

78. Thomas BA, Logar CM, Anderson AE. Renal replacement therapy in congestive heart failure requiring left ventricular assist device augmentation. Perit Dial Int 2012;32:386-92. 
Table S1 Mechanical circulatory support devices

\begin{tabular}{|c|c|c|c|c|c|c|c|c|}
\hline & \multirow{2}{*}{ IABP } & \multicolumn{2}{|c|}{ Percutaneous VAD } & \multirow{2}{*}{ VA-ECMO } & \multicolumn{4}{|c|}{ Surgical VAD } \\
\hline & & Impella & Tandem-Heart & & Centrimag & Abiomed & Heartmate II & HVAD \\
\hline Mechanism & Counter pulsation & Axial, continuous flow & Centrifugal, continuous flow & Centrifugal, continuous flow & $\begin{array}{l}\text { Centrifugal, } \\
\text { continuous flow }\end{array}$ & $\begin{array}{l}\text { Pneumatic, pulsatile } \\
\text { flow }\end{array}$ & $\begin{array}{l}\text { Rotary, continuous } \\
\text { flow }\end{array}$ & $\begin{array}{l}\text { Centrifugal, } \\
\text { continuous flow }\end{array}$ \\
\hline Support & LV support & LVAD; RVAD & LVAD; RVAD; BiVAD & LV/RV support; oxygenation & LVAD; RVAD; BiVAD & LVAD; RVAD; BiVAD & LVAD & VAD \\
\hline Effect on LV/LA & $\downarrow$ LV afterload & Unloads LV/RV & Unloads LA & $\uparrow L V$ afterload & Unloads LV & Directly unloads LV & Directly unloads LV & $\begin{array}{l}\text { Directly unloads } \\
\text { LV }\end{array}$ \\
\hline $\begin{array}{l}\text { Device-specific } \\
\text { potential issues }\end{array}$ & $\begin{array}{l}\text { Air emboli; malposition; aortic injury; } \\
\text { mesenteric thrombosis; } \downarrow \text { platelets }\end{array}$ & $\begin{array}{l}\text { Hemolysis; pump migration; AV injury; } \\
\text { tamponade; ventricular arrhythmias }\end{array}$ & $\begin{array}{l}\text { Cannula migration; tamponade; } \\
\text { emboli; inter-atrial shunt }\end{array}$ & $\begin{array}{c}\text { Circuit clotting; LV dilatation; } \\
\text { differential hypoxia (peripheral } \\
\text { VA-ECMO) }\end{array}$ & Blood or air emboli & $\begin{array}{l}\text { Limited mobility; } \\
\text { bleeding; sepsis }\end{array}$ & $\begin{array}{l}\text { Bleeding; sepsis; RV } \\
\text { failure }\end{array}$ & $\begin{array}{l}\text { Bleeding; sepsis; } \\
\text { RV failure }\end{array}$ \\
\hline $\begin{array}{l}\text { Device-specific } \\
\text { benefits }\end{array}$ & Easy, rapid insertion & Multiple platforms & Hemodynamic stability & $\begin{array}{l}\text { Rapid, bedside insertion; } \\
\text { oxygenation }\end{array}$ & $\begin{array}{l}\text { Extensive experience; } \\
\text { may add oxygenation }\end{array}$ & $\begin{array}{l}\text { Easy to use; } \\
\text { independent of } \\
\text { cardiac rhythm }\end{array}$ & $\begin{array}{l}\text { Easy insertion; small } \\
\text { size }\end{array}$ & $\begin{array}{l}\text { Thoracic insertion; } \\
\text { small size }\end{array}$ \\
\hline
\end{tabular}

\section{References}

79. Aneman A, Brechot N, Brodie D, et al. Advances in critical care management of patients undergoing cardiac surgery. Intensive Care Med 2018;44:799-810. 